\title{
L'ESPÉRANCE CHEZ LES PÈRES APOSTOLIQUES
}

Même si l'espérance, vertu théologale, est une des plus importantes conceptions de la théologie, l'évolution et la fluctuation de son contenu dans la tradition chrétienne pendant les siècles est assez peu explorét. Nous voudrions donc dans cet article examiner les deux paroles par lesquelles on a exprimé 1 'espérance: substantif $\varepsilon \lambda \pi \zeta_{S}$ et le verbe $\varepsilon \lambda \pi \zeta \zeta \omega$ chez les P’̀res Apostoliques, premiers témoins de cette trauition. Si nous avons un bon article sur le concept de l'espérance selon la Bible dans le Victionnaire de Kittel ${ }^{2}$, le contenu de ces deux paroles, assez fréquentes chez les pères Apostoliques/employé 56 fois: 37 comme substantif et 19 comme verbe/ ${ }^{3}$, jusqu’à présent n'était pas étudié.

Mais avant tout il faut faire quelques réserves. Les Pères Apostoliques ne voulaient pas donner de synthèse théologique. Dans leurs écrits, souvent occasionelles, ils expliquaient l'Evangile à des gens wodestes, dans un language simple, avec un but pratique: leur faire connaître la foi et les faire agir selon l'Evangile. De plus, nous avons repris et mis ensemble des textes clair semés dans leurs écrits, d'où langer que soit deforwée l'idée même de l'espérance. Eniin il faut se garder de chercher nos idées théologiques, riches d'une tralition bimillennaire, dans desécrits des premiers

1 Cfr M.J. Sicben, Voces. Eine Bibliographie zu wortern und Begriffen aus der Patristik/1918-1978/, Berlin 1980, 78 .

2 Gran Lessico del Nuovo Testamento fondato da G. Kittel, continuato da G. Friedrich, III, Brescia 1967, 507-548/édition allemande II $515-530 \%$ Cfr aussi Vocabulaire de théologie biblique, réd. Xavier Leon-Dufour, Paris $1970^{2}$. s.v. espérance.

3 H. Irraft, Clavis Patrum Apostolicorum. Konkordanz zu den Schrieten der Apostolischen Vater, Mitnchen 1963, 147-148. 
témoins de la Tradition. Nous levons tâcher de saisir leur mode de voir, souvent maladroit, polyvalent et mêie contradictoire.

Dans la première partie de cet article seront analysés tous les lieux où on rencontre ces leux termes dans le sens théologique; dans la seconde on cherchera à donner une synthèse théologique des résultats de la recherche.

\section{ANALYSE DES TEXTES}

\section{Didaché}

Dans la Didaché /I/II s./ une fois seulement on rencontrè le verbe $\varepsilon \lambda \pi \zeta \zeta \omega$, dans les célèbres "Deux Voies": "Tu ne commanderas pas avec aigreur à ton serviteur ou à ta servante, qui mettent leur

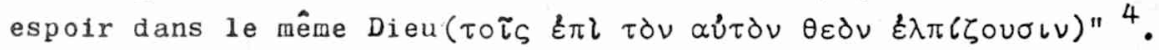
'E $\lambda \pi \iota \zeta \omega$ est $1 c i$ symonyme de "croire". La même phrase se trouve dans les "Deux Voies" insérées dans l'Epître de Barnabé5.

\section{Ignace d'Antioche}

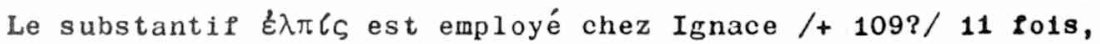
le verbe $\varepsilon \lambda \pi \zeta \zeta \omega-4$ lois.

Pour Ignace $I^{\prime}$ espérance a un charactère nettement christologique:

- Jésus est I'espérance parfaite ( $\eta \tau \varepsilon \lambda \varepsilon\left(\alpha \varepsilon \varepsilon_{\lambda} l_{\zeta \varsigma}\right)^{6}$;

- Jésus est commune espérance, au nom de laquelle 11 salue les

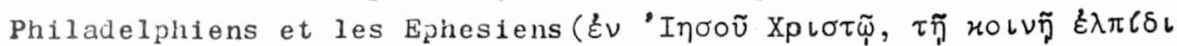
ग่น $\mu \tilde{\omega} \nu$ ?;

- Jésus est "notre espérance dont nous partagerons la vie [éternelle] si nous vivons maintenant en lui"8; "auquel nous espérons nous

4 Didache 4, 10, SCh 248, 162.

5 Epistula Barnabae 19,7, SCh 172, 204.

6 Ad Smyrnaeos 10, 2. J'al employé 1'édition de A. Lelong /Les Père! Apostoliques, III, Ignace d'Antioche et Polycarpe de Smyrne, Pariı $19272 /$, p. 92. On trouve aussi dans les manuscrits la forme "fol parfaite".

7 Ad Philadelphenos 11, 2; Ad Ejhesos 21, 2; c Pr Ad Philalelphenos 5, 2, Lelong pp. $80,26,72$.

8 Ad Trallianos 2, 2, Lelong p. 43. 
réunir par la résurrection" 9 ; "à laquelle /espérance/ Dieu veullle qu'aucun de vous ne soit jamais infidele" 10 .

- Jésus est I'espérance aninée par la charité dans une joie innocente

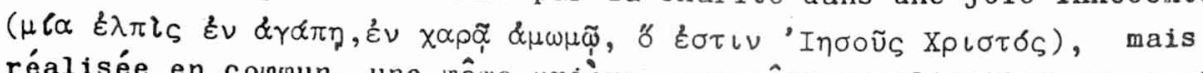
réalisée en commun, une nểe priére, une mêne supplication, un seul et même esprit ${ }^{11}$.

- Jésus est l’espérance nouvelle embrassée par ceux qui vivaient sous 1 'ancien ordre des choses ${ }^{12}$.

- Le Christ est $I^{\prime}$ objet de 1 'espérance et de $I^{\prime}$ attente des prophètes 13 et des chrétiens de Philadelphie, en qui ils ont mis leur espérance pour le corps, 1 âme et 1 'esprit par la foi, la charité, la concor$\mathrm{de} \mathrm{e}^{14}$.

Car I'Evangile de Jésus est l'Evangile d'une nouvelle espérance (

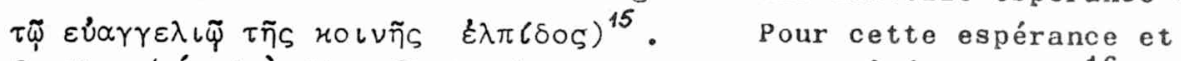
le Nom /c'est à dire Christ/ Ignace vient enchaîné le Syrie ${ }^{16}$.

Il faut prier sans cesse pour les autres hommes, car 11 faut une espérance qu’ 11 s reviendront à Dieu par la pénitence ( $\varepsilon \lambda \pi l \zeta$ $\mu \varepsilon \tau \alpha \nu \circ(\alpha \varsigma){ }^{17}$.

Enfin le verbe $\varepsilon_{\lambda} \lambda \zeta \zeta \omega$ est employé deux fols sans aucun sens theologique particulier 18 .

\section{Polycarpe}

Dans la lettre de Polycarpe, évêque des Smyrne /+ 155 ou 156/ aux Phillppiens, le mot $\varepsilon^{\prime} \lambda \iota_{S}$ n'est employé que deux fois.

"On doit avoir sans cesse les yeux attachés sur l'espérance ( $\tau$ j

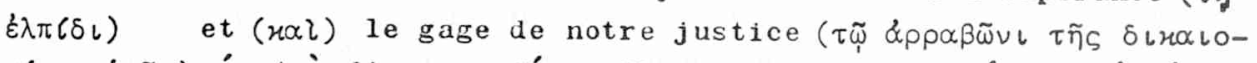

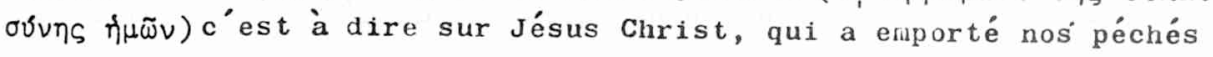

Ad Trallianos, inscriptio, Lelong p. 43.

10 Ad Magnesios 11 , Lelong p. 38.

11 Ioidem, 7, 1, Lelong p. 34.

12 Ibidem, 9, 1, Lelong p. 37.

13 Ad Philadelphenos 5, 2, Leiong p. 73.

14 Ibidem, 11, 2, Lelong p. 81 .

15 Ibidem, 5, 2, Lelong p. 74 .

16 Ad Ephesos 1, 2, Lelong p. 4.

17 Ibidem, 10, 1, Lelong p. 16.

18 Ad liomanos 1, 1, et Ad Ephesos 1, 1, Lelong pp. 56 et 4. 
sur son propre corps sur le bois"19. Si on prend $x \alpha \zeta$ dans 1 e sens explicatif, l'espérance sera le gage de notre salut - nous l'avons, car Jésus a detruit par la croix nos péchés.

Dans un autre fragment Polycarpe exhorte les Philippiens à méditer et relire les lettres de S. Paul, ainsi il seront apfermis dans la foi regue. "Cette foi qui est notre mère à tous, elle est

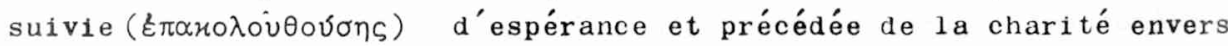
Dieu, envers le Christ et envers le prochain. Quiconque pratique ces

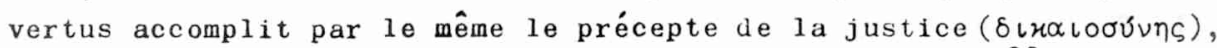
car quiconque possède la charité est loin de tout péché"20. Il est assez difficile de constater quelles sont, selon Polycarpe, les relations mutuelles entre ces trois vertus: est-ce-que la charité précède et la foi et l'espérance ou seulement l'espérance. En tout cas pour pouvoir accomplir la justice, 11 faut avoir l'espérance. S'il est difficile d'expliquer exactement la pensée de Polycarpe, nous constatons toutefois que dans ce texte apparaît pour la première fois dans la litterature post-apostolique le groupe des trois vertus bien differencié et doté de tout son dynamisme 21 .

\section{Clément de Rome}

Le substantif $\varepsilon \lambda \pi \zeta_{S}$ se trouve 5 fois dans la lettre aux Corinthiens de Clément de Rome /+101?/, le verbe $\varepsilon \lambda \pi \zeta \zeta \omega$ aussi 5 fois. L'espérance est liée à la foi: par le sang de l'Agneau seront sauvés tous ceux qui croient et espérent en Dieu, comme Rahab du livre de Josué22. Ces deux vertus sont mis ensemble dans un autre Praglient qui pose des problemes d'interprétation: "Par la vie de Dieu, par la vie du Selgneur Jésus Christ et de l'Esprit Saint - la

19 Ad Philippenses 8, 1, Lelong p. 119-121.

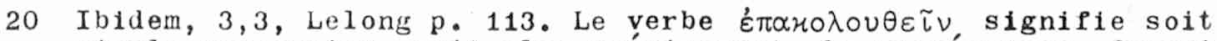
simple succession, soit plus précisement, la conséquence. On voit dans ce texte une ébauche de réplexion sur la connexion des vertus, déjà formulée par Clément d'Alexandrie: "les vertus s accompagnent les unes les autres"/Stromata 2, 18, 20/. Cfr J. Liebaert, Les enseignements moraux des Pères Apostoliques, Gembloux 1970, 75.

$21 \mathrm{~J}$. Liebaert, op. cit., 76.

22 Ad Corinthios 12, 7, SCh $167,120$. 
Poi et l'espérance des élus - celui qui aura pratiqué sans regret les préceptes et les commandements donnés par Dieu ... celui-là sera rangé et compté au nombre de ceux qui sont sauvés par Jésus Christ" 23. Nous ne sommes pas sûrs, si l'expression "la foi et l'espérance des élus" constitue une apposition à l'ensemble aux deux derniers, c'est à dire à Jésus et l'Esprit saint, ou, enfin, à l'Esprit saint seul. Il $y$ a, en tout cas, une certaine indentification de la foi et de 1 'espérance soit avec Trois Personnes Divines, soit avec Jésus et l'Esprit Saint, soit avec Ésprit Saint seul.

Dans l'espérance de la résurrection, nos âmes s'attachent à Dieu qui reste fidèle à ses promesses et juste dans ses jugenents ${ }^{24}$. En donnant 1 'exemple de Loth, clément oppose ceux qui espèrent en Dieu et ne seront pas délaissés et les rebelles de Corinthe qui seront punis ${ }^{25}$. Et c'est pourquoi les rebelles sont menacés d'être privés de $I^{\prime}$ espérance, qu'on ne trouve avec lumilité que dans

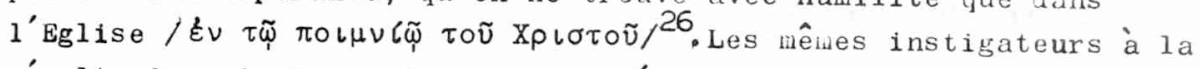
révolte devraient prendre en considération, quel est le fondernent

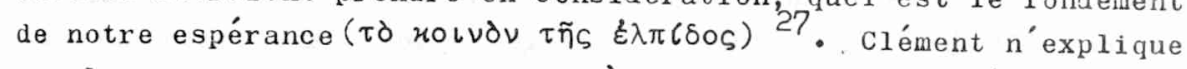
pas le sens exacte de cette dernière expression. Peut-êtrépourra-t-on le mettre au clair par le contexte. Il semble que pour clément le fondement de l'espérance est la charité et la crainte de Dieu( $\not \gamma d \pi \eta$,  confession des fautes ( $\xi \xi \circ \mu \circ \lambda \circ \gamma \varepsilon \tilde{\iota} \sigma \theta \alpha \iota)$. Trois fois clément cite des Pragments tirés de la Sainte Eeriture qui parlent de l'espérance 28.

23 Ibidem, 58, 2, SCh 167, 193-195.

24 Iuidem, 27, 1, SCh $167,144$.

25 Ibidem, 11, 1, SCh 167, 116.

26 Ibidem, 57, 2, SCh 167, 190. Cfr G.W.H. Lampe, A Patristic Greek Lexicon, Oxford 1961, 1110 .

27 Ibidem, 51, 1, SCh 167, 182. CPr W. Bauer, W 8rterbuch zum Neuen Testament, Berlin 19715, 866: "das, was d. Hoffnung gemeinsames hat, d. gemeinsame Grundlage der Hoffnung". 28 Ibidem, $16,16=\operatorname{Ps~} 21,7-9 ; 22,8=\operatorname{Ps} 31,10 ; 57,7=\operatorname{Prov} 01$,
33 , SCh $167,128,140,192$. 
5. L'homélie dite II Epître de Clément

Dans 1 'homélie du II $s_{\bullet}$, dite II Epître de S. Clément aux Corinthiens le substantif $\varepsilon \lambda \pi l_{s}$ est employé 2 fois, aussi que le verbe $\varepsilon \lambda \pi \iota \zeta \omega$.

En voyant les châtiments des condamnés, les justes "rendront gloire à leur Dieu, procluwant qu'il y a une espérance poúr qui a servi Dieu de tout son coeur"29. Il faut persévérer dans l'espérance pour obtenir la récompense, c'est à dire le salut ${ }^{30}$. Il n'y a d'espérance qu'en Dieu pour notre salut ${ }^{31}$. Car Jésus Christ est Dieu, "juge des vivants et des morts/Act. 10, 42/; et si nous estimons peu notre salut /.../ médiocre est notre espérance /... et nous somes en péchén 32 .

\section{Epître de Barnabé}

-Dans 1'Epître de Barnabé on trouve 7 fois le substantif $\varepsilon \lambda \pi \zeta_{S}$ et le verbe $\varepsilon \lambda \pi \zeta \zeta \omega$ fois. Comme nous le voyons, dans cette lettre ces deux paroles sont employées le plus eréquemment dans les écrits des Pères Apostoliques.

L'espérance est un des trois enseignements ( $\delta \delta \gamma \mu \alpha \tau \alpha)$ du Seigneur: "L'espérance de la vie ( $\zeta \omega \tilde{\eta} s \varepsilon \lambda(\varsigma)$, commencement et fin de notre foi

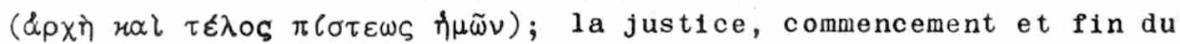
jugement; 1 'amour, l'attestation pleine de joie et d'allégresse des oeuvres accomplies dans la justicen ${ }^{33}$.

29 Epistula II Ps-Clementis ad Corinthios 17, 7. Nous avons employé la traduction française de S. Suzanne-Dominique/dans: Les écrits des Peres Apostollques, Paris $1962 /$ p. 131.

30 Ibidem, 11, 5, Suzanne-Dominique p. 125.

31 Ibilem, 1, 7, Suzanne-Dominique p. 116.

32 Ibidem, 1, 1, Suzanne-Dominique p. 115.

33 Epistula Barnabae 1, 6, SCh 172, 76. Pour 1'authenticité de ce texte cer ibidem, n. 2. En ce qui concerne le mot $\delta \delta \gamma \mu \alpha$ c fr G. W. H. Lampe, op.cit., 377: of precepts of Christian conducts. Sur ce texte voir aussi les observations de J. Liebaert, op.cit., 155: "Que l'espérance de la vie/éternelle/ soit mise en vedette est sans doute 1 ' Indice de l'influence d'une perspective fortement eschatologique; les trois "maximes'du reste sont marqués par cette perspective; vie éternellle,jugement, témoignage des oeuvres au jugement. L'idée que l'espérance est principe et fin'de la fol 
$L^{\prime}$ auteur de $l^{\prime E p} \hat{i} t r e$ souligne fortement $I^{\prime}$ interdépendence de l'espérance et de la fol. L'espérance de la vie est rondement de la foi. Ce lien nous le constatons dans plusieurs textes, par exemple dans les expressions: "par 1 'espérance de la pol en lul (

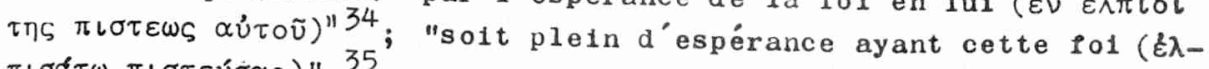
$\pi \iota \sigma \alpha \tau \omega \pi \iota \sigma \tau \varepsilon \cup ́ \sigma \alpha s) " 35$. Nous ne trouvons pas dans I'Epître une explication plus detalliéede cette lialson, mals seulement une ébauche dans deux textes: celui dejà cité où l'espérance de la vie est considérée comme commencement et fin le notre poi ${ }^{36}$, et dans une autre: "C'est pour moi, aussi une absolue nécessité de vous aimer plus que mon âme, car la fol et l'amour qui vous habitent sont grands, fondés sur l'espérance de la vie"37. Mais au contraire dans un autre texte la poi et 1 'amour sont considérés comme raison de la conversion qui porte l'espérance: "Toute parale de foi et d'anour qui sortira de votre bouche sera pour un grand nombre cause de conversion et d'espérance" ${ }^{38}$. Par la foi et 1 espérance on est scellé dans le baptême par 1'alliance de Jésus 39 .

L'auteur de l'Epître souligne très fortement le caractère christologique de I'espérance, car tout est en lui et pour lui 40 . Le Christ, plus justement, sa croix, était déjà objet d'espérance dans 1 'Ancien Testament à travers les types:

- le serpent d'airain /Nom. 21, 8/ - celui qui vient au serpent plein d'espérance avec la foi en son efficacité, sera sauvé ${ }^{4}$;

implique un lien très étroit entre les deux vertus et pratiquement leur assimilation".

34 Epistula Barnabae 4, 8, $\mathrm{SCh} 172,99$.

35 Ibidem, 12, 7, SCh 172, 170. CPr aussi 6, 2.

36 Cer ibidem, 31 .

37 Ibidem, 1, 4, SCh 172, 75.

38 Ibidem, 11, 7, SCh 172, 165.

39 I bidem, 4, 8, 'SCh 172, 98. Cfr infrä, Dans le texte de "Didache" déja cité /note 4 et 5/ la foi et 1 "éspérance sont des synonymes.

40 Ibidem, 12, 7, SCh 172, 170. Cfr Rom.11, 36; Col. 1, 16. L'espérance porte'sur le salut et la vie éternelie; eile se fonde sur le Christ sauveur: elle est "l espérance en Jésus" $/ 11$, 11; cer 6,$9 ; 8,5 ; 12,2$ n. $; 16,8 /$ et en, 1 a çrolx de Jésus $/ 11,8 /$, J. Liebąert, op.cit., 156. Ainsi 1'espérance que nous avons est eile "l'esperince en Jésus" - nous trouvons ici un christocentrisme trés vigoureusement affirné, 1bidem, 128.

41 Epistula Barnabae 12, 7, SCh 172, 170. 
- Molse est le type du Christ: en réalisant une préfiguration de la croix et de celui qui devait souffrir, car s'ils/les Israélites/ n'espèrent pas en lui, ils seront éternellement attaqués ${ }^{42}$; ils ne peuvent pas être sauvés à moins d'espérer en $14 i^{43}$; Moise a brisé les tables de l'Alliance pour que l'Alliance de Jésus fut scellée dans nos coeurs par l'espérance de la foi en lui ${ }^{44}$;

- la génisse rouge /Nom. 19/ - Barnabé trouve dans sa laine sur le bois le symbole de la croix et ceux qui espèrent en elle, vivront éternellement ${ }^{45}$.

Cette typologie christologique mène notre auteur à souligner contre les gnostiques/docètes/la valeur salvifique de la chair du Christ. L'espérance dans la croix n'aura de sens que si Jésus a un corps. Hoise, selon Barnabé, aurait dit: "Mettez votre espérance ... en Jésus, qui doit se révéler à vous dans la chair" ${ }^{46}$. Probablement un sens antidocète ont aussi les mots concernant les Juies qui ont cru au temple: "Celui qui croira en elle/en pierre/ vivra éternellement. Plagons - nous donc notre espérance en une pierre? Certes non. wais c'est que le seigneur a rendu forte sa chair"47.

Cet aspect cinristologique est souligné par le lien de l'espérance et du baptême, coinme son eruit ${ }^{48}$. Mais elle est aussi sa condition préalable: pour descendre dans l'eau, c'est à dire etre bâptisé,

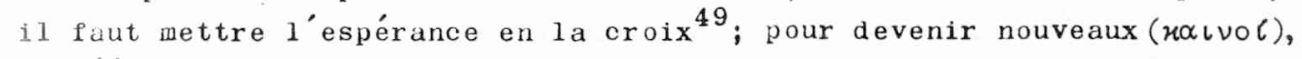

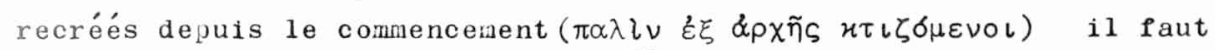
mettre notre espérance dans le Nom ${ }^{50}$. L'alliance de Jésus fut scellée

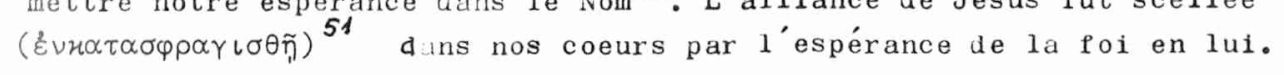

42 Ibidew, 12, 2, SCh $172,166$.

43 Ibidem, $12,3, \operatorname{SCh} 172,168 ; \operatorname{cor} 4,8$.

44 Ibidem, 4,8 , SCh $172,98$.

45 Ibider, 8, 5, SCh $172,140$.

46 Ibideil, 6, 9, SCh 172, 121-123.

47 Ibidem, 63, SCh 172, 117. CPr aussi 16, 1., Nous trouvons ici le lien dejámentioné, entre la foi et l'espérance.

48 Iòiden, 11, 11, SCh $172,166$.

49 Ibiden, $11,8, \operatorname{SCh} 172,164$.

50 Ibidem, 16, 8, SCh $172,192$.

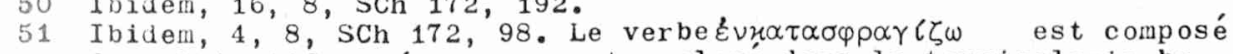
du substantif $\sigma \varphi p \not \gamma$ ig souvant employé dans la terminologie baptismale. Cfr G. W. H. Lampe, op. cit. 1356. 
On trouve encore chez Barnabé quelques textes d'une noindre 1mportance théologlque. Les Juifs ont mis leur espérance dans le teinple et c'est pourquoi leur espérance est vaine ${ }^{52}$. L'auteur espère

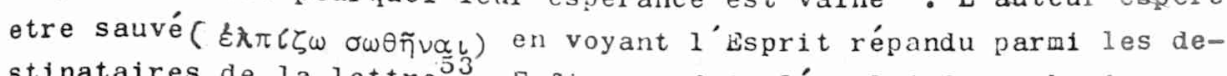
stinataires de la lettre ${ }^{5}$. Enfin une fois $l^{\prime}$ emploi du verbe $\varepsilon \lambda \pi \zeta \zeta \omega$ est théologiquement neutre ${ }^{54}$.

\section{Hermas}

Dans "Pasteur", I'oeuvre d'un ilermas, écrit probablement vers 130, le suistantif $\varepsilon \lambda \pi \zeta_{S}$ est employé 10 fois, le verbe $\varepsilon \lambda \pi \zeta \zeta \omega$ 5 rois.

Dans "Pasteur" la problématique de l'espérance se tourne autour du problème de la pénitence. Par le péché on perd l'espérance 55 , par la pénitence on la retrouve ${ }^{56}$. L'auteur énumère les catégories des personnes qui ont ou n'ont pas l'espérance d'être sauvées, mais rarement 11 donne des réflexions sur la nature de l'espérance mêne. Enumérant les dites catégories, il ajoute que pour ceux qui n'ont

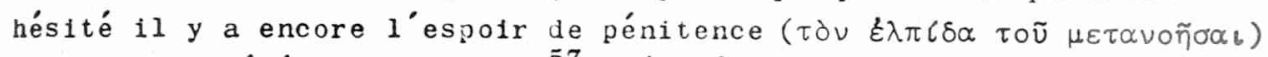
car ils ont été toujours bons ${ }^{57}$. L'espérance, selon lui, est une arme contre le diable, car il ne peut dominer les serviteurs de Dieu, si du fond du coeur 1 ls espèrent en $141^{58}$. Il y a des personnes qu1 ont 1 'espérance de la pénitence, autrenent dit du salut ( $\varepsilon \lambda \pi l s \mu \varepsilon \tau \alpha-$ vol $\alpha \varsigma, \mu \varepsilon \tau \alpha \nu \circ \tilde{\eta} \sigma \alpha \iota, \sigma \omega \theta \tilde{\eta} \nu \alpha \iota, \zeta \omega \tilde{\eta} \varsigma) 59$, ou il ne 1 'ont pas 60 . Un texte dans Pasteur est neutre théologiquement 61 .

52 Ibidew, 16, 1, SCh $172,188$.

53 Ibidea, 1, 2, SCh 172,72 .

54 Ividem, 17, 1 , SCh $172,194$.

55 Pastor, Visio $1,1,9 / 1,9 /$; Nandatum $5,1,7 / 33,7 /$; Similitudo $8,9,4 / 75,4 / ; 9,14,3 / 91,3 / ; 9,26,2 / 103,2 /, 5 C h$ 53 bis; $80 ; 164-166 ; 282-284 ; 322 ; 342$.

56 Ibidem, Similitudo $6,2,4 / 62,4 / ; 8,6,5 / 72,5 / ; 8,7,2$ $/ 73,2 / ; 8,10,2 / 76,2 /, \mathrm{SCh}_{1} 53,246,276,278,284$.

57 Ibidem, Similitudo $8,6,5 / 72,5 / . \mathrm{SCh} 53,276$.

58 Ibialem, Manàatum 12, 5, 2/48, 2/, SCh 53, 206.

59 Ibidem, Similitudo $6,2 / 62,4 / ; 8,5 / 72,5 / ; 8,2,9 / 68,9 / ; 8,7$ I $/ 73,7 / ; 8,9,4 / 75,4 / ; 8,11,2 / 77,2 / ; 9,14,3 / 91,3 /, \mathrm{SCh}$
$53,246,266,276,270,282,286,322$.

60 Ibidem, Visio $1,1,9 / 1,9 /$; Mandatum 5, $7 / 33,7 /$; Similitudo $9,26,2 / 103,2 /, \operatorname{SCh} 53,80,166,342$.

61 Ibldem, Visio $3,11,3 / 19,3 /, \mathrm{SCh} 53,128$. 


\section{SYNTHÈSE THÉOLOGIQUE}

Nous devons maintenant, l'analyse rafte, tirer des conclusions théologiques.

1. Il faut nettement distinguer deux sens du mot "espérance", employe parles Pères Apostoliques: disposition subjective d'un chrétien et une réalité objective - objet de l'espérance. Dans le premier sens, les Pères parlent de l'espérance comme d'une disposition de 1 'homme envers Dieu et le salut; dans l'autre, l'espérance c'est Jésus Christ même, la religion Chrétienne et l'Evangile qui porte 1 'espérance ${ }^{62}$.

2. Le Christ était déjà objet d'espérance dans I'Ancien Testament, pas directement mais à travers les types, comme 1 'affirme 1 'auteur de l'Epitre de Barnabé; en outre les personnages de l'Ancien Testament nous ont lonné l'exemple de l'espérance, comme Loth ou Rahaj. Mais, comme le souligne Ignace, dans ses lettres concentrées sur la personne du Christ, dans Jésus est la vraie espérance, nouvelle et parfaite, anirée par la charité dans une joie innocente. Jésus est le gage de notre justice. Nous espérons etre unis à lui après la résurrection.L'Evangile de Jésus est l'Evangile de l'espérance. Jésus, objet de notre espérance, est Dieu incarné, qui s'est révélé dans la chair - cet accent antidocète est mis clairenent en refiep par Barnabé, et moins clairement par Polycarpe; on peut s'etonner de ne pas le trouver chez Ignace, nettement antidocète. Nous pouvons dire que pour les Pères Apostoliques l'espérance est christocentrique: la vie, la résurrection, le salut, les biens à venir se retrouvent dans le Christ, vrai sujet de l'espérance.

3. L'espérance est liée avec le baptême. Elle le précède: pour descenare dans l'eau, il raut mettre son espérance dans la croix et dans le Now de Jésus, comme nous le dit Barnabé. Mais elle est aussi le fruit du baptême et elle scelle dans les ânes l'alliance avec Jésus. Nous entrons dans 1 eau couverts de péchés et nous en sortons

62 J. Liebaert, op.cit., 59. 
devenus nouveaux avec la crainte de Dieu dans nos coeurs et avec 1 'espérance dans 1 'esprit.

4. L'espérance est liée avec la pénitence - cette verité est particulièrement développée par Hermas. Le péché detruit l'espérance, dans la pénitence on la retrouve; par elle on obtient l'espérance du salut et de la vie éternelle. Elle est une arwe contre le diable qui n'a pas accès à ceux, qui l'ont dans leur coeur.

5. Il est assez difficile de définir l'espérance telle qu'elle est exprimée dans les écrits des pères Apostoliques, mais ce n'étalt pas leur but. L'espérance est un don, en premier lieu liée avec la foi et la charité, puis avec les autres vertus. Le lien avec les vertus théologales nous mène à l'enseignenent de S. Paul sur la trlad des vertus / 1 Thes. 1, 2; 1 Cor. 13, 13; Gal. 5, 5/. Les Pères Aposto liques présentaient une interdépendance des vertus. L'espérance est

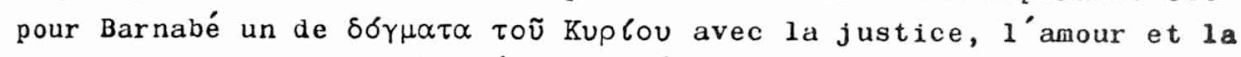
poi. Il souligne aussi une étroite dépendance de la foi et de l'espérance, parlant d'unc espérance dans la foi et de l'espérance de la foi. Dans les "Deux Voies" on va encore plus loin, on ilentifie la fol et l'espérance. Siais il est difficile de définir plus exactement ces relations selon les Pères Apostoliques. L'espérance est un don de la Trinité ou du Saint Esprit et elle trouve son accomplissement dans la communauté de l’Eglise.

Je crois que les Peres Apostoliques nous proposent quelques thèmes à méditer et à approfondire:

- Le Christ - notre espérance;

- l'espérance comie fruit et condition des sacrements et, particulièrement de la pénitence;

- conditionnement de la foi par l'espérance et de l'espérance par la foi. 